$12-17-2021$

\title{
The Relationship Between Discoid Meniscus and Articular Cartilage Thickness: A Quantitative Observational Study With MRI
}

Joseph Brutico

Thomas Jefferson University

Margaret Wright

Thomas Jefferson University

Sarah Kamel

Thomas Jefferson University

Adam C Zoga

Thomas Jefferson University

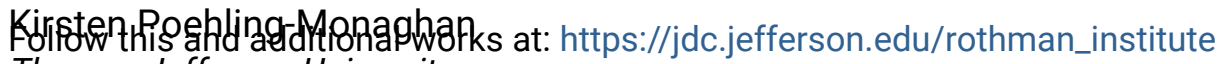
Thomas Jefferson University

Part of the Orthopedics Commons

\section{Let us know how access to this document benefits you See next page for additional authors}

\section{Recommended Citation}

Brutico, Joseph; Wright, Margaret; Kamel, Sarah; Zoga, Adam C; Poehling-Monaghan, Kirsten; and Hammoud, Sommer, "The Relationship Between Discoid Meniscus and Articular Cartilage Thickness: A Quantitative Observational Study With MRI" (2021). Rothman Institute Faculty Papers. Paper 154.

https://jdc.jefferson.edu/rothman_institute/154

This Article is brought to you for free and open access by the Jefferson Digital Commons. The Jefferson Digital Commons is a service of Thomas Jefferson University's Center for Teaching and Learning (CTL). The Commons is a showcase for Jefferson books and journals, peer-reviewed scholarly publications, unique historical collections from the University archives, and teaching tools. The Jefferson Digital Commons allows researchers and interested readers anywhere in the world to learn about and keep up to date with Jefferson scholarship. This article has been accepted for inclusion in Rothman Institute Faculty Papers by an authorized administrator of the Jefferson Digital Commons. For more information, please contact: JeffersonDigitalCommons@jefferson.edu. 


\section{Authors}

Joseph Brutico, Margaret Wright, Sarah Kamel, Adam C Zoga, Kirsten Poehling-Monaghan, and Sommer Hammoud 


\title{
The Relationship Between Discoid Meniscus and Articular Cartilage Thickness
}

\section{A Quantitative Observational Study With MRI}

\author{
Joseph M. Brutico, ${ }^{\star}$ BS, Margaret L. Wright, ${ }^{*}$ MD, Sarah I. Kamel, ${ }^{\dagger}$ MD, Adam C. Zoga, ${ }^{\dagger}$ MD, \\ Kirsten Poehling-Monaghan, ${ }^{*} \mathrm{MD}$, and Sommer Hammoud, ${ }^{* \ddagger} \mathrm{MD}$ \\ Investigation performed at Rothman Institute, Philadelphia, Pennsylvania, USA
}

\begin{abstract}
Background: Several cadaveric imaging studies have demonstrated that the articular cartilage thickness on the tibial plateau varies depending on coverage by native meniscal tissue. These differences are thought to partially contribute to the rates of cartilage degeneration and development of osteoarthritis after meniscectomy. Because there is greater tibial plateau coverage with meniscal tissue in the setting of a discoid meniscus, these findings may also have implications for the long-term health of the knee after saucerization of a torn discoid meniscus.
\end{abstract}

Purpose: To evaluate the relationship between lateral compartment articular cartilage thickness and the presence or absence of a discoid meniscus.

Study Design: Cross-sectional study; Level of evidence, 3.

Methods: Included in the study were 25 patients younger than 40 years of age who had undergone a 1.5-T or 3-T knee magnetic resonance imaging (MRI) between 2010 and 2016 at a single institution and had an intact, lateral discoid meniscus. Only patients with an otherwise asymptomatic lateral compartment were included. The authors then identified 35 agematched controls with a nondiscoid, intact lateral meniscus who underwent knee MRI at the same institution and during the same period. The articular cartilage thicknesses in 6 zones of the lateral femoral condyle (LFC) and lateral tibial plateau (LTP) were measured for each patient by 2 musculoskeletal radiologists, and the mean thicknesses were compared between the study and control groups.

Results: The average age at MRI was 22.63 years (range, 8.30-35.90 years) for the discoid group and 20.93 years (range, 8.4334.99 years) for the nondiscoid group. The nondiscoid group had significantly greater mean articular cartilage thickness in all 6 zones of the LTP $(P<.05$ for all). When comparing the zones of the LFC, there was no significant difference in the mean thickness in any zone between the 2 groups.

Conclusion: Patients with discoid menisci had thinner baseline articular cartilage thickness in the LTP compared with those patients without discoid menisci.

Keywords: discoid meniscus; articular cartilage thickness; lateral femoral condyle; lateral tibial plateau

Several cadaveric and advanced-imaging studies analyzing articular cartilage thickness of the tibia have demonstrated thicker cartilage in areas uncovered by meniscus. ${ }^{17}$ Such variations in native cartilage thickness further impact the patterns and rates of cartilage degeneration and development of knee osteoarthritis after meniscectomy. ${ }^{10,14,15}$ Therefore, in theory, patients with a discoid lateral meniscus, defined as medialized extent of the meniscus, may have thinner native articular cartilage on their tibial plateau due to overcoverage by meniscal tissue.

The Orthopaedic Journal of Sports Medicine, 9(12), 23259671211062258 DOI: $10.1177 / 23259671211062258$

(c) The Author(s) 2021
Considering that the menisci are important load distributors and shock absorbers, preservation of meniscal tissue during partial meniscectomy is an important consideration during surgical management of a meniscal tear in a young patient. Treatment of a symptomatic and/or torn discoid meniscus with saucerization, and exposure of the underlying joint surface to previously unseen contact pressures, may also have implications for accelerated subsequent cartilage degeneration in these patients. ${ }^{10-12,14,19}$ In their case series, Lee et $\mathrm{al}^{13}$ noted progression of articular cartilage degeneration in symptomatic patients who had undergone partial meniscectomy for a torn discoid lateral meniscus after an average follow-up period of 6.8 years. These results are supported by those of Ahn et al, ${ }^{1}$ who found a positive

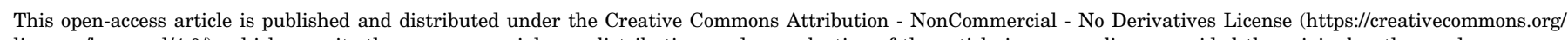

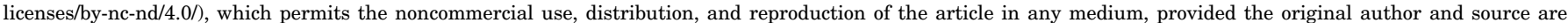

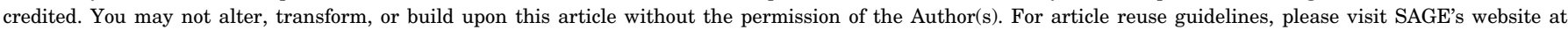
http://www.sagepub.com/journals-permissions. 

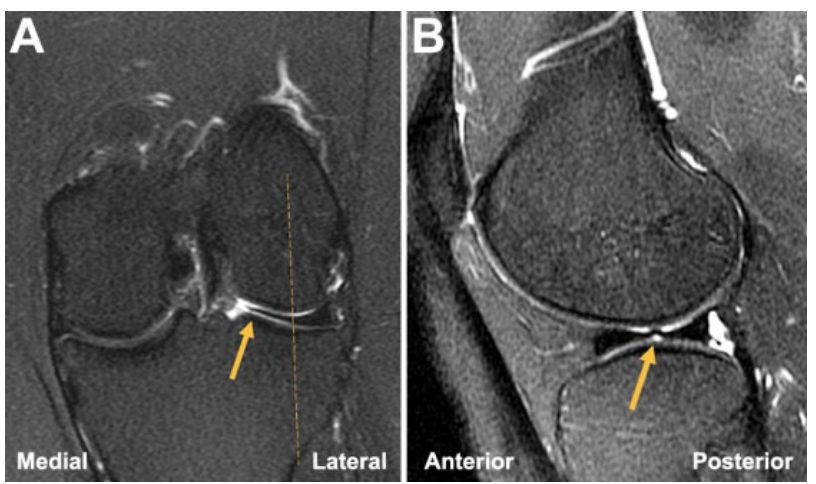

Figure 1. A 25-year-old female patient with a discoid lateral meniscus. (A) On a coronal T2-weighted fat-saturated image obtained at the anteroposterior midpoint of the lateral tibial plateau, a discoid meniscus is seen measuring $20 \mathrm{~mm}$ in transverse width, extending medially beyond the apex of the lateral femoral condyle (dashed line). The medial-most extent of the discoid lateral meniscus is annotated by the arrow. (B) On sagittal T2-weighted imaging, the anterior and posterior horns of a discoid meniscus should be continuous (arrow) on at least 3 or more 5-mm slices; in this case, it was seen on 4 contiguous slices.

correlation between the relative percentage of discoid lateral meniscal thickness and the formation of KellgrenLawrence grade 3 or 4 osteoarthritis after partial meniscectomy. However, it is unknown whether the propensity of patients with a discoid meniscus to develop early arthritic changes is primarily due to changes in the contact pressures seen by the articular surface after meniscectomy, or if these patients are also at increased risk because of thinner native articular cartilage surfaces. To date, the relationship between the presence of a discoid meniscus and lateral compartment articular cartilage thickness has not been extensively studied.

The purpose of this study was to compare the articular cartilage thickness of the lateral tibiofemoral weightbearing articular surfaces in patients with an intact discoid and nondiscoid lateral meniscus using magnetic resonance imaging (MRI) to better understand the potential implications of treatment of discoid menisci.

\section{METHODS}

After receiving institutional review board approval, we identified a consecutive series of patients younger than 40 years of age who underwent a noncontrast, nonarthrogram knee
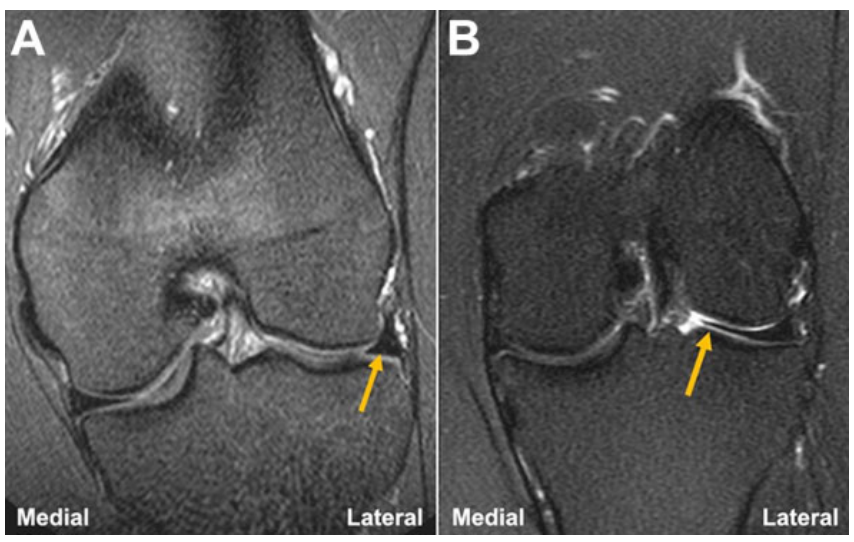

Figure 2. Coronal T2 fat-saturated sequences demonstrating the 3 study groups. (A) Normal lateral meniscus

MRI between 2010 and 2016. All patients with an intact discoid lateral meniscus in an otherwise asymptomatic lateral compartment were included in the study. The presence of lateral discoid meniscus on MRI was defined as a medialized extension of the inner margin of the meniscus beyond the midline apex of the lateral femoral condyle (LFC) (Figure 1). While all patients initially underwent MRI for a suspected knee injury, those with lateral knee pain due to fracture, chondrosis, osseous stress response, or high-grade ligament sprains were excluded. A group of age- and sex-matched controls were also identified, defined as those with a normal knee MRI and symptoms that did not involve the lateral compartment of the knee. Controls with fracture, chondrosis, osseous stress response, or high-grade ligament sprains in the lateral compartment were excluded. All MRI was performed on $\mathrm{a} \geq 1$.5-T system using a vendor-specific quadrature transmit/receive coil (4-channel or 8-channel). A standard knee protocol was performed for all studies, among which sequences of interest to our study were the axial, sagittal, and coronal fat-saturated T2 fast spin echo sequences highlighting the water content of articular cartilage. All knee examinations were interpreted by 2 musculoskeletal fellowship-trained radiologists (A.C.Z., S.I.K.).

Patients meeting study criteria were grouped according to their meniscal morphology on MRI: normal/nondiscoid versus discoid (Figure 2).

All cartilage measurements of the weightbearing lateral tibiofemoral compartment were performed in the craniocaudal dimension on the sagittal T2-weighted fast spin echo sequence. To ensure consistent and reproducible

${ }^{\ddagger}$ Address correspondence to Sommer Hammoud, MD, Rothman Orthopaedics at Thomas Jefferson University, 925 Chestnut Street, Philadelphia, PA 19107 (email: Sommer.Hammoud@RothmanOrtho.com).

*Rothman Institute, Thomas Jefferson University, Philadelphia, Pennsylvania, USA.

${ }^{\dagger}$ Thomas Jefferson University Hospital, Philadelphia, Pennsylvania, USA.

Final revision submitted August 1, 2021; accepted August 24, 2021.

One or more of the authors has declared the following potential conflict of interest or source of funding: S.H. has received education payments from Arthrex and hospitality payments from Smith \& Nephew. AOSSM checks author disclosures against the Open Payments Database (OPD). AOSSM has not conducted an independent investigation on the OPD and disclaims any liability or responsibility relating thereto.

Ethical approval for this study was obtained from Thomas Jefferson University. 

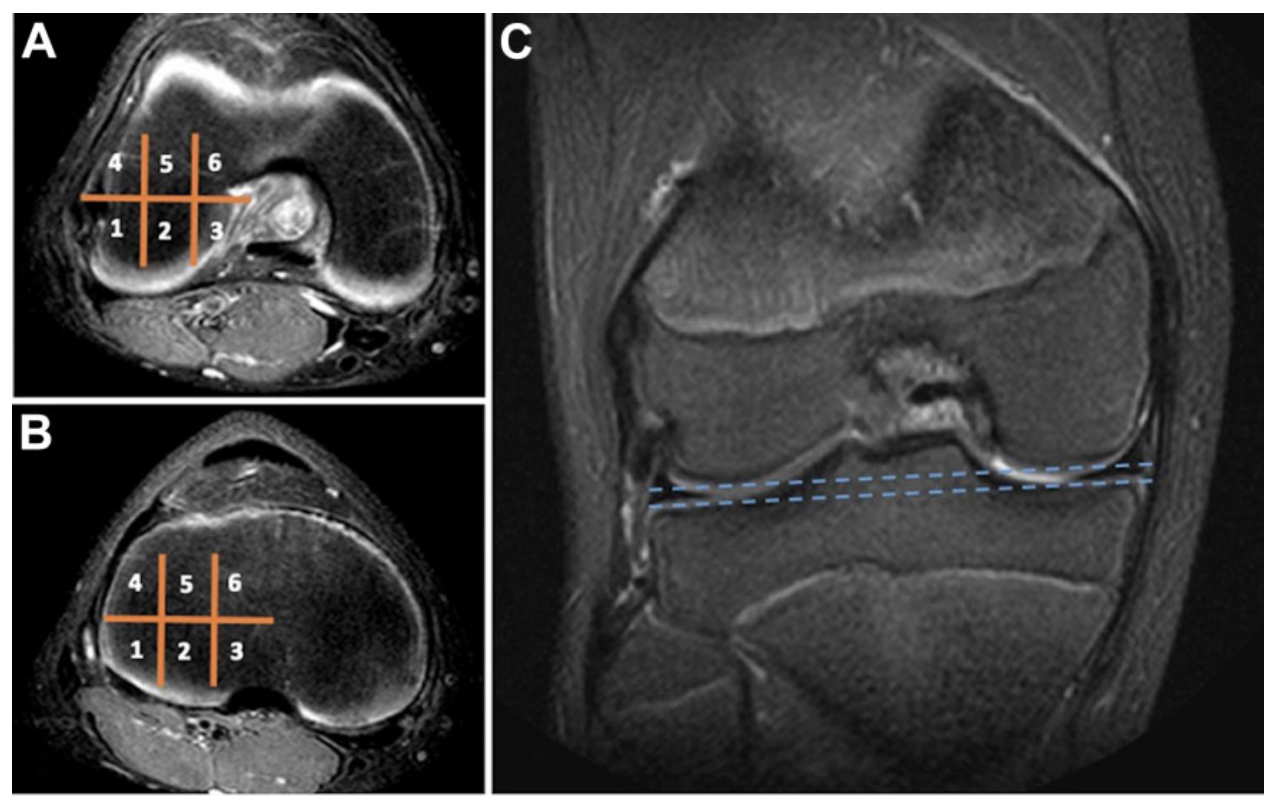

Figure 3. T2-weighted fat-saturated axial sequences through the weightbearing (A) lateral femoral condyle articular cartilage and (B) lateral tibial plateau as annotated on (C) a cross-referenced coronal T2 fat-saturated sequence. A 3-column by 2-row grid with 6 zones, as shown, was overlaid on the weightbearing articular cartilage surfaces to systematically compare measurements of cartilage thickness across the study population.

measurements of cartilage thickness, the lateral tibial plateau (LTP) and the weightbearing LFC cartilage were divided into 6 equally sized zones using a 3-column by 2 -row grid (Figure 3 ).

Scout lines were utilized to cross-reference the coronal and axial planes and obtain measurements of cartilage thickness in each zone, reported in millimeters. The 6 zone measurements for each weightbearing lateral articular surface were then pooled. Descriptive statistics, including the mean, range, and standard deviation, were calculated. The Student $t$ test was used to compare continuous variables among the 2 groups. The chi-square test was used for categorical data. All $P$ values less than .05 were considered statistically significant. Interrater reliability between the 2 radiologists was determined by calculating the intraclass correlation coefficient (ICC). The ICC scores were interpreted as follows: 0 to 0.49 , weak reliability; 0.50 to 0.74 , moderate reliability; and 0.75 to 1.00 , strong reliability. ${ }^{12}$ Interrater reliability was evaluated for each zone of both the LTP and LFC.

\section{RESULTS}

Thirty-five patients with a lateral discoid meniscus on MRI were identified. Ten $(28.6 \%)$ patients within the discoid group had a tear of their lateral meniscus and were excluded from the study. Thirty-five age-matched patients with a normal, nondiscoid meniscus were used as controls. There was no significant difference with respect to sex $(P=.609)$ and mean age at the time of MRI $(P=.667)$ in each group. Patient data for each group can be found in Table 1.
TABLE 1

Patient Data According to Meniscal Morphology ${ }^{a}$

\begin{tabular}{lccc}
\hline & $\begin{array}{c}\text { Discoid Meniscus } \\
(\mathrm{n}=25)\end{array}$ & $\begin{array}{c}\text { Nondiscoid Meniscus } \\
(\mathrm{n}=35)\end{array}$ & $P$ \\
\hline Sex & & & .609 \\
$\quad$ Male & $8(32.0)$ & $13(37.1)$ & \\
$\quad$ Female & $17(68.0)$ & $22(62.9)$ & \\
$\begin{array}{c}\text { Age at } \\
\text { MRI, y }\end{array}$ & $22.63 \pm 7.75(8.30-35.90)$ & $20.93 \pm 7.67(8.43-34.99)$ & .667 \\
\hline
\end{tabular}

${ }^{a}$ Data are presented as mean $\pm \mathrm{SD}$ (range) or $\mathrm{n}(\%)$. MRI, magnetic resonance imaging.

The mean thickness of the LTP articular cartilage was significantly greater in each zone in the nondiscoid group compared with the discoid group (Table 2). The ICC between each musculoskeletal radiologist was determined to be strong for each individual zone of the LTP.

Regarding the LFC (Table 3), comparison of articular cartilage thickness of the control group and discoid group did not elucidate any statistically significant differences in any zone. The ICC between each musculoskeletal radiologist was determined to be moderate-strong for each individual zone of the LFC.

The discoid meniscus group was further categorized based on morphology using the Watanabe classification. ${ }^{21}$ Fourteen patients were found to have complete discoid menisci, 9 had incomplete discoid menisci, and the remaining 2 patients had Wrisberg variants. There was no significant association between discoid meniscal morphology and articular cartilage thickness $(P=.868)$. 
TABLE 2

A Comparison of the Articular Cartilage Thickness of the Lateral Tibial Plateau Between the Discoid and Nondiscoid Groups ${ }^{a}$

\begin{tabular}{|c|c|c|c|c|c|}
\hline \multirow[b]{2}{*}{ Cartilage Zone } & \multicolumn{5}{|c|}{ Cartilage Thickness, mm } \\
\hline & Discoid Meniscus & Nondiscoid Meniscus & $P$ & ICC $(95 \% \mathrm{CI})$ & Agreement \\
\hline 1 & $1.25 \pm 0.47(0.60-2.30)$ & $1.65 \pm 0.73(0.90-3.80)$ & .012 & $0.922(0.718-0.980)$ & Strong \\
\hline 2 & $1.55 \pm 0.61(0.80-2.70)$ & $1.98 \pm 0.88(0.70-3.80)$ & .029 & $0.977(0.913-0.994)$ & Strong \\
\hline 3 & $1.48 \pm 0.65(0.70-3.60)$ & $2.41 \pm 1.34(0.70-5.60)$ & .001 & $0.955(0.838-0.988)$ & Strong \\
\hline 4 & $1.24 \pm 0.46(0.60-2.50)$ & $1.59 \pm 0.76(0.60-3.80)$ & .031 & $0.979(0.865-0.995)$ & Strong \\
\hline 5 & $1.37 \pm 0.56(0.80-3.20)$ & $1.80 \pm 0.89(0.60-4.00)$ & .024 & $0.892(0.612-0.972)$ & Strong \\
\hline 6 & $1.41 \pm 0.73(0.60-4.40)$ & $2.22 \pm 1.36(0.90-5.60)$ & .004 & $0.906(0.585-0.977)$ & Strong \\
\hline
\end{tabular}

${ }^{a}$ Data are presented as mean \pm SD (range) unless otherwise indicated. Bolded $P$ values indicate a statistically significant difference between groups $(P<.05)$. The intraclass correlation coefficient (ICC) between each independent radiologist was determined to be strong (ICC, >0.75).

TABLE 3

A Comparison of the Articular Cartilage Thickness of the Lateral Femoral Condyle Between the Discoid and Nondiscoid Groups $^{a}$

\begin{tabular}{|c|c|c|c|c|c|}
\hline \multirow[b]{2}{*}{ Cartilage Zone } & \multicolumn{5}{|c|}{ Cartilage Thickness, mm } \\
\hline & Discoid Meniscus & Nondiscoid Meniscus & $P$ & ICC $(95 \% \mathrm{CI})$ & Agreement \\
\hline 1 & $1.96 \pm 0.64(0.90-3.30)$ & $2.06 \pm 0.60(1.20-3.90)$ & .513 & $0.807(0.425-0.948)$ & Strong \\
\hline 2 & $2.38 \pm 0.96(0.90-4.40)$ & $2.27 \pm 0.80(1.30-4.00)$ & .656 & $0.937(0.778-0.984)$ & Strong \\
\hline 3 & $2.11 \pm 0.83(0.90-4.40)$ & $2.32 \pm 0.92(1.30-5.30)$ & .362 & $0.511(0.000-0.844)$ & Moderate \\
\hline 4 & $1.36 \pm 0.46(0.60-2.80)$ & $1.61 \pm 0.54(0.70-2.80)$ & .062 & $0.945(0.796-0.986)$ & Strong \\
\hline 5 & $1.60 \pm 0.67(0.50-3.60)$ & $1.73 \pm 0.71(0.90-4.40)$ & .507 & $0.896(0.636-0.973)$ & Strong \\
\hline 6 & $1.52 \pm 0.71(0.50-3.50)$ & $1.65 \pm 0.59(0.50-3.50)$ & .439 & $0.828(0.471-0.954)$ & Strong \\
\hline
\end{tabular}

${ }^{a}$ Data are presented as mean \pm SD (range) unless otherwise indicated. The intraclass correlation coefficient (ICC) between each independent radiologist was determined to be at least moderate (ICC, $>0.50$ ).

\section{DISCUSSION}

To our knowledge, this is currently the largest study to evaluate the differences in lateral compartment articular cartilage thickness between patients with and without discoid menisci. Our results confirm that patients with a discoid meniscus are likely to have thinner articular cartilage in the LTP when compared with controls. Prior studies have demonstrated that decreased loading is associated with thinner articular cartilage surfaces, and in this case, it is likely that the larger and/or thicker discoid meniscal stress shields LTP during weightbearing and results in the development of thinner hyaline cartilage in that compartment. While the difference was statistically significant in all 6 zones of the tibial plateau, the difference in average thickness was greatest in the 2 more central cartilage zones, which supports the fact that the medialized coverage of the discoid lateral meniscus contributes to relatively thinner articular cartilage in this location. Notably, no significant differences were found when comparing the articular cartilage thickness of the LFC between the groups, which is in accordance with data from other studies ${ }^{3,17}$ and suggests that meniscal coverage is not associated with femoral condylar cartilage thickness. This may be because of the larger and more variable areas of weightbearing on the femoral condyles as the knee tracks through flexion and extension, therefore rendering differences in meniscal coverage less consequential to the ultimate thickness of the femoral articular surface.

Only 1 previous study ${ }^{18}$ has examined the relationship between articular cartilage thickness and meniscal coverage, and it found no difference in the femoral and tibial articular cartilage thickness between patients with normal and discoid menisci in both the sagittal and coronal planes. However, that study was smaller than the present study and measured only cartilage thickness at the midpoint of the lateral compartment in the coronal plane and at the midpoint of the discoid meniscus in the sagittal plane for each patient. As such, it is therefore difficult to compare the cartilage thickness measurements in their study to those in our study. At least 4 other studies ${ }^{3,6,7,15}$ have examined the articular cartilage thickness of the LTP and LFC in patients with normal menisci. Of these studies, Li et $\mathrm{al}^{15}$ implemented the most similar style of measurement, in which the articular cartilage of the LTP and femoral condyle was measured using a 9-zone and a 12-zone methodology, respectively. Their measurements of the LTP (mean range, 1.4-4.0 $\mathrm{mm}$ ) and $\mathrm{LFC}$ (mean range, 1.7-2.9 $\mathrm{mm}$ ) were most similar to those of the discoid group in our study, which demonstrates the consistency of this measurement system and its potential ability to detect small differences in cartilage thickness if they exist. 
The meniscus is an important structure for load bearing, stabilization, and pressure distribution during ambulation, and disruption to the normal kinematics and pressure distribution of the knee after lateral meniscectomy can predispose patients to early osteoarthritis. ${ }^{2,4,5,8,15,20,22}$ Longo et $\mathrm{al}^{16}$ found that the prevalence of osteoarthritis in the lateral tibiofemoral compartment is $58 \%$ after partial meniscectomy (follow-up range, 5.1-12.1 years), and Lee et $\mathrm{al}^{14}$ found that $84 \%$ of children with discoid menisci developed early arthritic changes after partial and subtotal/total meniscectomies at an average of 4.3 years. These studies demonstrate the significance of the lateral meniscus for stress distribution in both normal and discoid knees and underscore the importance of reserving meniscal saucerization for symptomatic patients only, because of the relatively short period in which these knees can develop arthritic changes after meniscectomy. Because patients who are being treated for symptomatic discoid menisci are often young, understanding the various factors that may contribute to the onset or rapid progression of arthritic changes in their knees is paramount. Percent meniscal resection, lower extremity alignment, discoid meniscal thickness, tear pattern, and duration of symptoms have all been proposed as factors that may be related to the progression of arthritic changes. ${ }^{9,13}$ Based on the results of the present study, the articular cartilage thickness of the LTP at the time of meniscectomy may also be different between patients with and without discoid menisci, which may be an additional factor to consider in the progression of arthritic changes in these patients. While the thickness of the articular cartilage cannot be changed at the time of surgical treatment, a very gradual increase in weightbearing and limitation of athletic activities to low-impact sports may help the articular surface to accommodate previously unseen contact pressures and better preserve the baseline cartilage thickness in these patients.

More challenging to study than articular cartilage thickness is the quality and mechanical properties of the cartilage itself. It is possible that the biomechanical and histologic properties of articular cartilage are also different between patients with discoid menisci and normal menisci, as the cartilage is somewhat shielded from stress by the discoid meniscus during growth and development. Future studies may seek to examine the differences in the structure and biomechanical properties of articular cartilage in patients with and without discoid menisci, as well as the potential for articular cartilage to adapt to increased contact pressure after discoid saucerization, to further understand the development of arthritic changes in these patients.

This study does have a few limitations. First, the small sample size of the groups may not have allowed for detection of differences in cartilage thickness among all of the groups if one existed, although this is the largest study of its type to date. In addition, it is unknown whether the statistically significant differences in cartilage thickness contribute to a clinical difference in the progression of arthritic changes in this patient population. Regarding measurement technique, the researchers involved in cartilage measurement could not be blinded to group identity, although every effort was taken to measure each patient consistently, and the measurements were performed with high-quality software. One possible limitation is that all imaging was obtained at $1.5 \mathrm{~T}$. While imaging parameters were standardized among included patients, there may be less variability among the measurements of cartilage thickness than if imaging was acquired at a higher-field-strength magnet with improved resolution, although the ICC for most measurements in this study was high. In addition, advanced cartilage mapping was not performed, and therefore, the quality of cartilage between the 2 groups could not be compared. Finally, the patients were matched by age and sex, but this may not have completely controlled for factors like patient size, hip-knee angle, genetics, activity level, and degree of skeletal maturity, which also could have affected their cartilage stresses and/or thickness.

\section{CONCLUSION}

The risk for development of osteoarthritic changes after surgical treatment of a symptomatic discoid lateral meniscus is well-known. Our study indicates that patients with discoid menisci also have thinner native articular cartilage of the LTP compared with patients with a normal lateral meniscus. As a result, baseline articular cartilage thickness underlying the discoid meniscus may also be a risk factor for the progression of osteoarthritis in patients with discoid menisci.

\section{REFERENCES}

1. Ahn JH, Kang DM, Choi KJ. Risk factors for radiographic progression of osteoarthritis after partial meniscectomy of discoid lateral meniscus tear. Orthop Traumatol Surg Res. 2017;103(8): 1183-1188.

2. Andriacchi TP, Dyrby CO. Interactions between kinematics and loading during walking for the normal and ACL deficient knee. J Biomech. 2005;38(2):293-298.

3. Ateshian GA, Soslowsky LJ, Mow VC. Quantitation of articular surface topography and cartilage thickness in knee joints using stereophotogrammetry. J Biomech. 1991;24(8):761-776.

4. Bergfeld JA, McAllister DR, Parker RD, Valdevit ADC, Kambic HE. A biomechanical comparison of posterior cruciate ligament reconstruction techniques. Am J Sports Med. 2001;29(2):129-136.

5. Boynton MD, Tietjens BR. Long-term followup of the untreated isolated posterior cruciate ligament-deficient knee. Am J Sports Med. 1996;24(3):306-310.

6. Cohen ZA, McCarthy DM, Kwak SD, et al. Knee cartilage topography, thickness, and contact areas from MRI: in-vitro calibration and in-vivo measurements. Osteoarthritis Cartilage. 1999;7(1):95-109.

7. Eckstein F, Winzheimer M, Hohe J, Englmeier KH, Reiser M. Interindividual variability and correlation among morphological parameters of knee joint cartilage plates: analysis with three-dimensional MR imaging. Osteoarthritis Cartilage. 2001;9(2):101-111.

8. Gill TJ, DeFrate LE, Wang C, et al. The biomechanical effect of posterior cruciate ligament reconstruction on knee joint function: kinematic response to simulated muscle loads. Am J Sports Med. 2003; 31(4):530-536.

9. Higuchi H, Kimura M, Shirakura K, Terauchi M, Takagishi K. Factors affecting long-term results after arthroscopic partial meniscectomy. Clin Orthop Relat Res. 2000;(377):161-168. 
10. Kim JG, Han SW, Lee DH. Diagnosis and treatment of discoid meniscus. Knee Surg Relat Res. 2016;28(4):255-262.

11. Kim SJ, Chun YM, Jeong JH, Ryu SW, Oh KS, Lubis AMT. Effects of arthroscopic meniscectomy on the long-term prognosis for the discoid lateral meniscus. Knee Surg Sports Traumatol Arthrosc. 2007; 15(11):1315-1320.

12. Koo TK, Li MY. A guideline of selecting and reporting intraclass correlation coefficients for reliability research. J Chiropr Med. 2016;15(2): 155-163.

13. Lee CR, Bin SI, Kim JM, Kim NK. Magnetic resonance imaging findings in symptomatic patients after arthroscopic partial meniscectomy for torn discoid lateral meniscus. Arthroscopy. 2016;32(11):2366-2372.

14. Lee DH, Kim TH, Kim JM, Bin SI. Results of subtotal/total or partial meniscectomy for discoid lateral meniscus in children. Arthroscopy. 2009;25(5):496-503.

15. Li G, Park SE, DeFrate LE, Schutzer ME, Ji L, Gill TJ, Rubash HE. The cartilage thickness distribution in the tibiofemoral joint and its correlation with cartilage-to-cartilage contact. Clin Biomech. 2005;20(7): 736-744.

16. Longo UG, Ciuffreda M, Candela V, et al. Knee osteoarthritis after arthroscopic partial meniscectomy: prevalence and progression of radiographic changes after 5 to 12 years compared with contralateral knee. J Knee Surg. 2019;32(05):407-413.

17. Maher SA, Wang H, Koff MF, Belkin N, Potter HG, Rodeo SA. Clinical platform for understanding the relationship between joint contact mechanics and articular cartilage changes after meniscal surgery. J Orthop Res Off Publ Orthop Res Soc. 2017;35(3):600-611.

18. Oni DB, Jeyapalan $\mathrm{K}$, Oni OOA. An observational study on MR images of the effect of the discoid meniscus on articular cartilage thickness. Knee. 2011;18(3):202-204.

19. Räber DA, Friederich NF, Hefti F. Discoid lateral meniscus in children. Long-term follow-up after total meniscectomy. J Bone Joint Surg Am. 1998;80(11):1579-1586.

20. Roos H, Adalberth T, Dahlberg L, Lohmander LS. Osteoarthritis of the knee after injury to the anterior cruciate ligament or meniscus: the influence of time and age. Osteoarthritis Cartilage. 1995;3(4):261-267.

21. Watanabe M, Takeda S, Ikeuchi H. Atlas of Arthroscopy: Masaki Watanabe, Sakae Takeda, Hiroshi lkeuchi. 3rd ed. Igaku-Shoin; 1979.

22. Yoo JD, Papannagari R, Park SE, DeFrate LE, Gill TJ, Li G. The effect of anterior cruciate ligament reconstruction on knee joint kinematics under simulated muscle loads. Am J Sports Med. 2005;33(2): 240-246. 